STRUCTURAL BIOLOGY COMMUNICATIONS

ISSN 2053-230X

Received 7 April 2020

Accepted 31 July 2020

Edited by G. G. Privé, University of Toronto, Canada

Keywords: membrane-protein purification; crystallization contaminant; transcription termination factor; cryoEM.

PDB reference: $E$. coli transcription termination factor Rho, 6wa8

Supporting information: this article has supporting information at journals.iucr.org/f
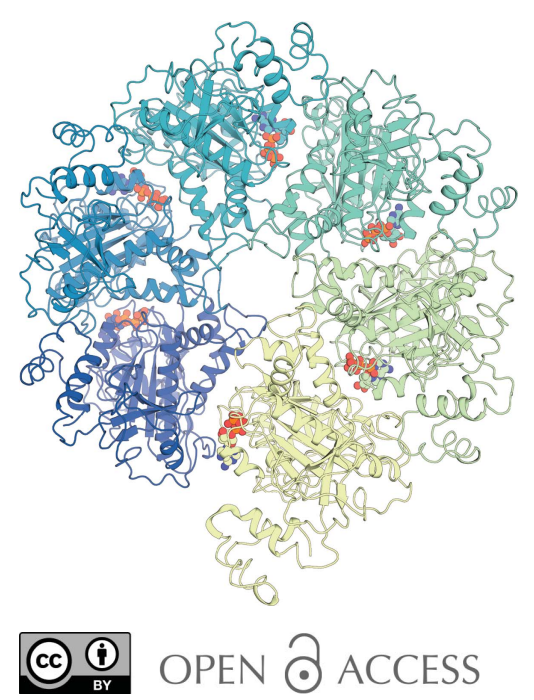

\section{Crystal structure of the Escherichia coli transcription termination factor Rho}

\author{
Chengcheng Fan ${ }^{\mathrm{a}}$ and Douglas C. Rees ${ }^{\mathrm{a}, \mathrm{b} *}$ \\ a Division of Chemistry and Chemical Engineering, California Institute of Technology, 1200 East California Boulevard, \\ Pasadena, CA 91125, USA, and ${ }^{\mathbf{b}}$ Howard Hughes Medical Institute, California Institute of Technology, 1200 East \\ California Boulevard, Pasadena, CA 91125, USA. *Correspondence e-mail: dcrees@caltech.edu
}

During the crystal structure analysis of an ATP-binding cassette (ABC) transporter overexpressed in Escherichia coli, a contaminant protein was crystallized. The identity of the contaminant was revealed by mass spectrometry to be the Escherichia coli transcription terminator factor Rho, structures of which had been previously determined in different conformational states. Although Rho was present at only $\sim 1 \%$ of the target protein (a bacterial homolog of the eukaryotic $\mathrm{ABC}$ transporter of mitochondria from Novosphingobium aromaticivorans; $\mathrm{NaAtm} 1)$, it preferentially crystallized in space group $C 2$ as thin plates that diffracted to $3.30 \AA$ resolution. The structure of Rho in this crystal form exhibits a hexameric open-ring staircase conformation with bound ATP; this characteristic structure was also observed on electronmicroscopy grids of the $\mathrm{NaAtm} 1$ preparation.

\section{Introduction}

One of the challenges in the crystallography of membrane proteins is their typically low expression level, which necessitates a significant degree of purification to separate the protein of interest from all other cellular proteins. This can consequently lead to the inadvertent purification of contaminant proteins that might otherwise be present at negligible levels when the target protein expresses at high levels. In unfortunate cases, these impurities may crystallize more readily than the target protein, leading to misplaced enthusiasm until the contaminant is recognized. As examples, the multi-drug efflux pump AcrB is a well known crystallization contaminant in membrane-protein preparations owing to its relatively high expression level during recombinant protein expressions with antibiotic selection and its nonspecific binding to Ni-NTA columns (Veesler et al., 2008; Das et al., 2007). Bacterioferritin has also been crystallized as a contaminant in preparations of cytochrome $c b b_{3}$ oxidase (Nam et al., 2010). In addition, exogenous proteins such as DNase, lysozyme and various proteases used in target protein purification have also been shown to be crystallization contaminants (Niedzialkowska et al., 2016). Compilations facilitate the identification of crystals of contaminant proteins (Hungler $e t$ al., 2016; Simpkin et al., 2018), but the crystallization of 'novel' impurities is still a concern. In this work, we report the crystal structure of a previously unreported contaminating protein, the transcription termination factor Rho from Escherichia coli, which was obtained during the structural analysis of a bacterial ATP-binding cassette (ABC) transporter. 
Rho is a hexameric RNA helicase that functions in transcription termination in E. coli. The six subunits together form a ring-like structure, and the structure switches between an open-ring staircase conformation and a closed-ring conformation coupled to the binding and hydrolysis of ATP (Skordalakes \& Berger, 2003; Thomsen \& Berger, 2009). Here, we present the crystal structure of Rho in an open-ring staircase conformation at $3.30 \AA$ A resolution with ATP bound.

\section{Materials and methods}

\subsection{Macromolecule production}

Rho copurified with the bacterial ABC exporter $\mathrm{NaAtm} 1$, which is a homolog of the $\mathrm{ABC}$ transporter of mitochondria (Atm1) from Novosphingobium aromaticivorans, using an E. coli expression system and a modified version of a previously published protocol (Lee et al., 2014). Briefly, frozen E. coli cell pellets containing overexpressed $\mathrm{NaAtm} 1$ (and Rho) were lysed in lysis buffer consisting of $100 \mathrm{mM} \mathrm{NaCl}$, $20 \mathrm{~m} M$ Tris pH 7.5, $40 \mathrm{~m} M$ imidazole $\mathrm{pH} 7.5,10 \mathrm{~m} M \mathrm{MgCl}_{2}$, $0.5 \%(w / v) n$-dodecyl- $\beta$-D-maltopyranoside (DDM; Anatrace), $0.5 \%(w / v)$ octaethylene glycol monododecyl ether (C12E8; Anatrace) with the addition of lysozyme, DNase and protease inhibitor. After stirring for $3 \mathrm{~h}$ at $4^{\circ} \mathrm{C}$, the lysate was subjected to ultracentrifugation at $\sim 113000 \mathrm{~g}$ for $45 \mathrm{~min}$ at $4^{\circ} \mathrm{C}$. The supernatant was collected, loaded onto a pre-washed Ni-NTA column in buffer consisting of $100 \mathrm{mM} \mathrm{NaCl}, 20 \mathrm{~m} M$ Tris $\mathrm{pH}$ 7.5, $50 \mathrm{~m} M$ imidazole $\mathrm{pH} 7.5,0.05 \% \mathrm{DDM}, 0.05 \% \mathrm{C} 12 \mathrm{E} 8$ and eluted with the same buffer containing $350 \mathrm{~m} M$ imidazole $\mathrm{pH}$ 7.5. The protein was further purified by size-exclusion chromatography (SEC) using a HiLoad 16/60 Superdex 200 column (GE Healthcare) with SEC buffer consisting of $100 \mathrm{~m} M \mathrm{NaCl}, 20 \mathrm{~m} M$ Tris $\mathrm{pH} 7.5,0.05 \%$ DDM, $0.05 \%$ C12E8. Peak fractions were collected and concentrated to $\sim 20 \mathrm{mg} \mathrm{ml}^{-1}$ using a $100 \mathrm{kDa}$ cutoff Amicon Ultra 15 concentrator (Millipore). Macromolecule-production information is summarized in Table 1.

\subsection{Crystallization}

Rho crystallized during the crystallization trials of $\mathrm{NaAtm} 1$ under optimized conditions based on MemGold (Molecular Dimensions) condition No. 68 at $20^{\circ} \mathrm{C}$ by hanging-drop vapor diffusion. The final crystallization condition consisted of $100 \mathrm{~m} M \mathrm{NaCl}, 100 \mathrm{~m} M$ Tris $\mathrm{pH} 8.3,28 \%$ polyethylene glycol 2000 monomethyl ether (PEG 2000 MME), $0.2 M$ nondetergent sulfobetaine 221 (NDSB-221), $20 \mathrm{~m} M$ ATP pH 7.5. The crystallization sample was prepared in $1 \mathrm{~m} M$ ATP $\mathrm{pH} 7.5$, $5 \mathrm{~m} M$ EDTA pH 7.5 in the presence and absence of $5 \mathrm{~m} M$ oxidized glutathione (GSSG) $\mathrm{pH} 7.5$, which is a transport ligand for NaAtm1. Thin plate-shaped crystals appeared in about two weeks. The crystals were harvested in cryoprotectant solution consisting of $100 \mathrm{mM} \mathrm{NaCl}, 100 \mathrm{~m} M$ Tris $\mathrm{pH} 8.3$, 28\% PEG 2000 MME with PEG 400 at $10 \%, 15 \%$ and $20 \%$ before flash-cooling in liquid nitrogen. Crystallization information is summarized in Table 2.
Table 1

Macromolecule-production information.

The protein was expressed in E. coli from the native promotor without the use of an expression plasmid.

\begin{tabular}{|c|c|}
\hline Source organism & E. coli \\
\hline DNA source & Genomic DNA from E. coli \\
\hline Expression host & E. coli BL21-Gold (DE3) \\
\hline \multirow{15}{*}{$\begin{array}{l}\text { Complete amino-acid sequence } \\
\text { of the construct produced }\end{array}$} & MNLTELKNTPVSELITLGENMGLENLARMR \\
\hline & KQDI IFAILKQHAKSGEDIFGDGVLEIL \\
\hline & QDGFGFLRSADSSYLAGPDDIYVSPSQI \\
\hline & RRFNLRTGDTISGKIRPPKEGERYFALL \\
\hline & KVNEVNFDKPENARNKILFENLTPLHAN \\
\hline & SRLRMERGNGSTEDLTARVLDLASPIGR \\
\hline & GQRGLIVAPPKAGKTMLLQNIAQSIAYN \\
\hline & HPDCVLMVLLIDERPEEVTEMQRLVKGE \\
\hline & VVASTFDEPASRHVQVAEMVIEKAKRLV \\
\hline & EHKKDVIILLDSITRLARAYNTVVPASG \\
\hline & KVLTGGVDANALHRPKRFFGAARNVEEG \\
\hline & GSLTIIATALIDTGSKMDEVIYEEFKGT \\
\hline & GNMELHLSRKIAEKRVFPAIDYNRSGTR \\
\hline & KEELLTTQEELQKMWILRKI IHPMGEID \\
\hline & AMEFLINKLAMTKTNDDFFEMMKRS \\
\hline
\end{tabular}

Table 2

Crystallization.

\begin{tabular}{ll}
\hline Method & Hanging-drop vapor diffusion \\
Plate type & Hampton Research VDX \\
Temperature $(\mathrm{K})$ & 293 \\
Protein concentration $\left(\mathrm{mg} \mathrm{ml}^{-1}\right)$ & $<1$ \\
Buffer composition of protein & $100 \mathrm{~m} M \mathrm{NaCl}, 20 \mathrm{~m} M$ Tris pH 7.5, \\
$\quad$ solution & $1 \mathrm{~m} M$ ATP \\
Composition of reservoir solution & $100 \mathrm{~m} M$ NaCl, 100 m $M$ Tris pH 8.3, \\
& $20 \mathrm{~m} M$ ATP, 200 m $M$ NDSB-221, \\
Volume and ratio of drop & $28 \%$ PEG 2000 MME \\
& $1 \mu \mathrm{l}$ protein solution $+1 \mu$ reservoir \\
Volume of reservoir $(\mu \mathrm{l})$ & solution \\
\hline
\end{tabular}

\subsection{Data collection and processing}

Crystals were screened on the GM/CA beamline 23-ID-B at the Advanced Photon Source (APS) and on beamline 12-2 at the Stanford Synchrotron Radiation Laboratory. The final data set was collected on the GM/CA beamline 23-ID-B with an EIGER 16M detector (Dectris) using JBluIce-EPICS (Stepanov et al., 2011), processed and integrated with $X D S$ (Kabsch, 2010) and scaled with AIMLESS (Evans \& Murshudov, 2013). The crystals of Rho diffracted to about $3.30 \AA$ resolution in space group $C 2$, with unit-cell parameters $a=161.8, b=101.9, c=184.0 \AA, \beta=107.8^{\circ}$. Data-collection and processing statistics are summarized in Table 3 .

\subsection{Structure solution and refinement}

The self-rotation function was calculated with the $C C P 4$ program MOLREP (Winn et al., 2011). Molecular replacement was performed with Phaser in Phenix (Liebschner et al., 2019) using a monomeric subunit of a previous structure of Rho with PDB code 1pvo (Skordalakes \& Berger, 2003) as a model. Initial jelly-body refinements were carried out with REFMAC5 in CCP4 (Winn et al., 2011). Subsequent iterative refinement and model-building runs were separately conducted with phenix.refine (Liebschner et al., 2019) and Coot (Emsley et al., 2010), respectively. The refined coordinates 
Table 3

Data collection and processing.

Values in parentheses are for the outer shell.

\begin{tabular}{ll}
\hline Diffraction source & GM/CA 23-ID-B, APS \\
Wavelength $(\AA)$ & 0.9793 \\
Temperature $(\mathrm{K})$ & 100 \\
Detector & EIGER 16M \\
Crystal-to-detector distance $(\mathrm{mm})$ & 400 \\
Rotation range per image $\left(^{\circ}\right)$ & 0.2 \\
Total rotation range $\left(^{\circ}\right)$ & 360 \\
Exposure time per image $(\mathrm{s})$ & 0.2 \\
Space group & $C 2$ \\
$a, b, c(\AA)$ & $161.75,101.90,184.02$ \\
$\alpha, \beta, \gamma\left({ }^{\circ}\right)$ & $90,107.8,90$ \\
Mosaicity $\left(^{\circ}\right)$ & 0.11 \\
Resolution range $(\AA)$ & $39.79-3.30(3.42-3.30)$ \\
Total No. of reflections & $304610(29798)$ \\
No. of unique reflections & $43031(4465)$ \\
Completeness $(\%)$ & $99.9(99.7)$ \\
Multiplicity & $7.1(6.7)$ \\
$\langle I / \sigma(I)\rangle$ & $6.6(1.5) \dagger$ \\
$R_{\text {p.i.m. }} \dagger$ & $0.103(0.729)$ \\
Overall $B$ factor from Wilson plot $\left(\AA^{2}\right)$ & $79.1 \ddagger$
\end{tabular}

$\dagger$ Overall resolution cutoff determined by data completeness and $\mathrm{CC}_{1 / 2}>0.50$ in the high-resolution shell. $I / \sigma(I)$ falls below 2.0 past $3.46 \AA$ resolution. $\neq$ There were ice rings in the data.

and structure factors have been deposited in the RCSB Protein Data Bank as entry 6wa8. Refinement statistics are summarized in Table 4.

\subsection{Electron-microscopy sample preparation and data processing}

The expression plasmid for the membrane-scaffolding protein MSP1D1 was purchased from Addgene (plasmid No. 20061). The expression and purification of MSP1D1 were carried out using published protocols with minor modifications (Ritchie et al., 2009). Reconstitution of NaAtm1 (and the Rho contaminant) with MSP1D1 was carried out with 1-palmitoyl-2-oleoyl-glycero-3-phosphocholine (POPC) at a 1:2:130 molar ratio of NaAtm1:MSP1D1:POPC. This reconstituted sample was incubated overnight at $4{ }^{\circ} \mathrm{C}$. After two hours of incubation, BioBeads were added at $200 \mathrm{mg} \mathrm{ml}^{-1}$ for detergent removal. The sample was then subjected to sizeexclusion chromatography on a Superdex 200 Increase 10/300 column (GE Healthcare). The peak fractions were pooled and concentrated to $\sim 8 \mathrm{mg} \mathrm{ml}^{-1}$.

EM grids were prepared using a protein concentration of $4 \mathrm{mg} \mathrm{ml}^{-1}$ in the presence of $5 \mathrm{~m} M$ GSSG and $5 \mathrm{mM}$ AMPPNP. $3 \mu$ protein sample was applied onto freshly glowdischarged QuantiFoil $\mathrm{Cu}$ R2/2 300 mesh grids and blotted for $4 \mathrm{~s}$ with a blot force of 0 and $100 \%$ humidity at room temperature using a Vitrobot Mark IV (FEI). Data were collected on a $200 \mathrm{keV}$ Talos Arctica with a Falcon III detector

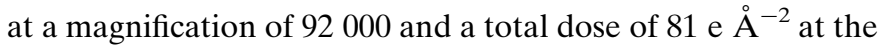
Caltech CryoEM Facility.

Data processing was performed with cryoSPARC2 (Punjani et al., 2017), following motion correction with full-frame motion and estimation of the contrast transfer function (CTF) with CTFFIND (Rohou \& Grigorieff, 2015). Particles were picked using a reconstruction of $N a A t m 1$ as a template and
Table 4

Structure solution and refinement.

Values in parentheses are for the outer shell.

\begin{tabular}{ll}
\hline Resolution range $(\AA)$ & $38.51-3.30(3.42-3.30)$ \\
Completeness $(\%)$ & $99.6(97.3)$ \\
$\sigma$ Cutoff & $8.9(1.3)$ \\
No. of reflections, working set & $40807(3928)$ \\
No. of reflections, test set & $2095(217)$ \\
Final $R_{\text {cryst }}$ & $0.252(0.320)$ \\
Final $R_{\text {free }}$ & $0.296(0.364)$ \\
No. of non-H atoms & \\
$\quad$ Total & 19776 \\
$\quad$ Protein & 19590 \\
$\quad$ Ligand & 186 \\
R.m.s. deviations & \\
$\quad$ Bond lengths $(\AA)$ & 0.002 \\
$\quad$ Angles $\left({ }^{\circ}\right)$ & 0.58 \\
Average $B$ factors $\left(\AA^{2}\right)$ & 101.8 \\
$\quad$ Overall & 101.4 \\
Protein & 143.2 \\
$\quad$ Ligand & \\
Ramachandran plot & 95.5 \\
$\quad$ Most favored $(\%)$ & 4.2 \\
Allowed $(\%)$ & 6 wa 8 \\
PDB entry &
\end{tabular}

extracted in cryoSPARC2. The initial 2D classification revealed a single class of Rho with $\sim 2500$ particles. The particles were then 2D-classified again into five classes, with four good classes with a total of $\sim 2200$ particles, as shown in Fig. 4(b).

\section{Results and discussion}

Crystals of Rho were unexpectedly obtained during studies of the bacterial ABC transporter NaAtm1 from $N$. aromaticivorans. NaAtm1 was recombinantly expressed in E. coli BL21Gold (DE3) cells with a C-terminal $6 \times$ His tag following a previously established protocol (Lee et al., 2014). After solubilization of the $E$. coli cells in DDM and C12E8, protein purification proceeded by Ni-NTA affinity purification followed by size-exclusion chromatography (Fig. 1a). SDSPAGE gels indicated a high degree of purity, although in subsequent analysis of overloaded gels a small amount of another protein was present at a molecular weight of $\sim 40 \mathrm{kDa}$ (Fig. 1b).

The crystals obtained during the crystallization optimization belonged to space group $C 2$, with unit-cell parameters $a=161.8, b=101.9, c=184.0 \AA, \beta=107.8^{\circ}$. The asymmetric unit volume was of sufficient size to accommodate an NaAtm1 dimer (molecular weight of $133 \mathrm{kDa}$ ). Analysis of the selfrotation function calculated from the diffraction data revealed a noncrystallographic symmetry (NCS) sixfold axis offset $\sim 3-4^{\circ}$ from the crystallographic $a$ axis. Interaction of the perpendicular twofold and sixfold axes generates a set of noncrystallographic twofold rotation operations separated by $60^{\circ}$ in the plane perpendicular to the NCS sixfold axis (Fig. 1c). Given the unit-cell dimensions, this apparent NCS was incompatible with dimeric $N a A t m 1$, which raised the possibility that a contaminant had crystallized. With an estimated molecular weight of $\sim 40 \mathrm{kDa}$ based on the observation of a 
faint impurity band in the gel and a Matthews coefficient analysis, we performed molecular replacements with known crystallization contaminants (Hungler et al., 2016; Simpkin et al., 2018), which all failed to yield a molecular-replacement solution.

The identification of Rho (molecular weight $47 \mathrm{kDa}$ ) was established by a mass-spectrometric analysis of the peptides prepared by trypsin digestion of the protein in the SDS-PAGE bands. Using this information, we were able to obtain a molecular-replacement solution using Rho in the AMPPNPbound state (PDB entry 1pvo; Skordalakes \& Berger, 2003) as the input model. The molecular-replacement results established that in these crystals Rho adopts a six-membered broken-staircase conformation. The structure was refined to an $R_{\text {work }}$ and $R_{\text {free }}$ of $25.2 \%$ and $29.6 \%$, respectively (Table 4 ).
The electron-density map also revealed ATP to be bound in all six subunits (Fig. 2a).

The individual Rho subunits are structurally similar overall, except for the terminal subunit at the 'break' in the staircase, where the first 50 residues at the $\mathrm{N}$-terminus exhibited a shift of 2-8 $\AA$ relative to the other monomers (Fig. $2 b$ ). The r.m.s.d. between the present structure and the molecular-replacement input model (PDB entry 1pvo) is $2.5 \AA$ using the $\mathrm{C}^{\alpha}$ positions for the superposition and $3.3 \AA$ when using all atoms of the six subunits in the hexamer. The r.m.s.d.s between individual subunits in the present structure and PDB entry 1pvo are 0.59-0.73 $\AA$, reflecting their similar tertiary structures. The relationship between adjacent subunits in the broken staircase of the present structure may be approximated by a screw operation with a rotation around the screw axis of $60.5^{\circ}$ per

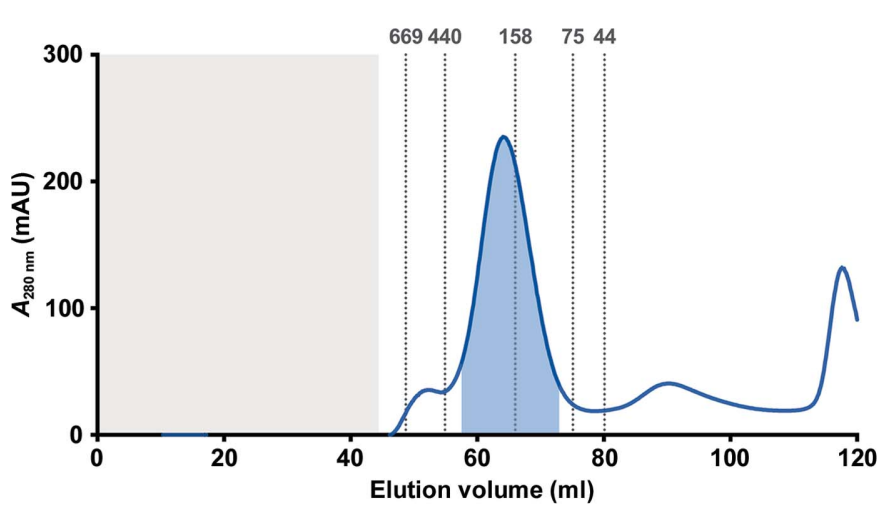

(a)

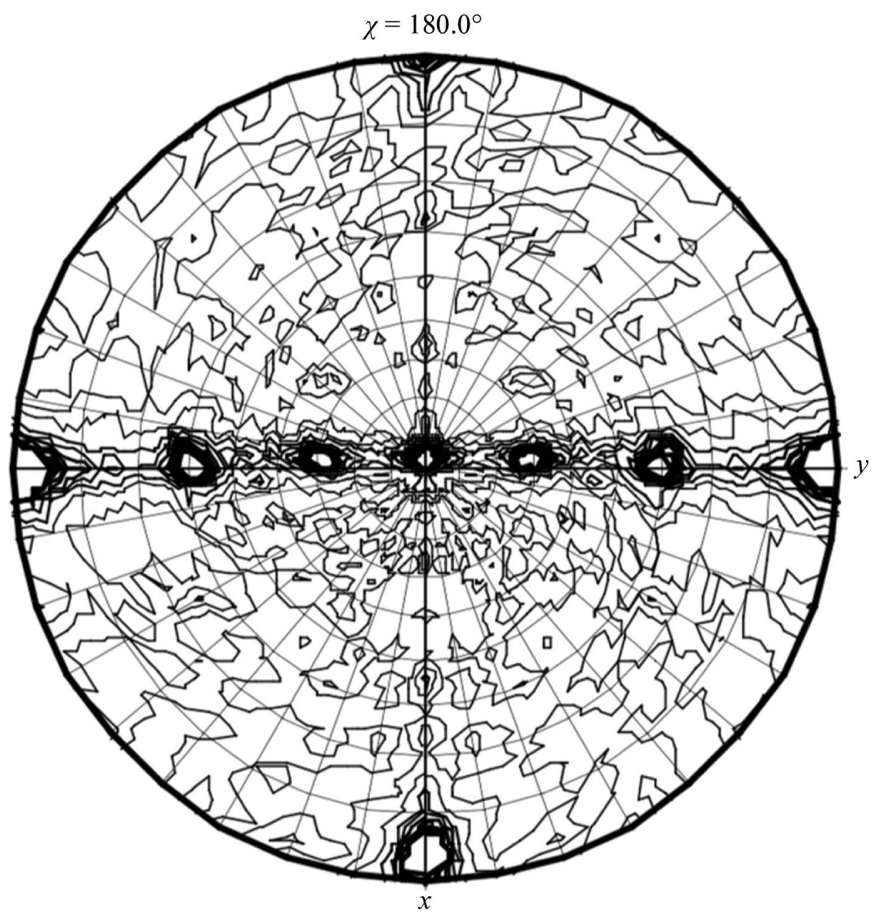

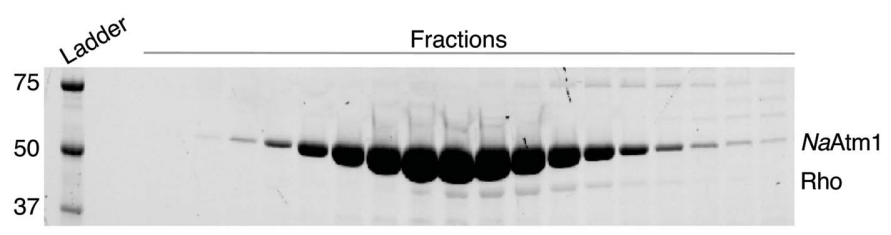

(b)

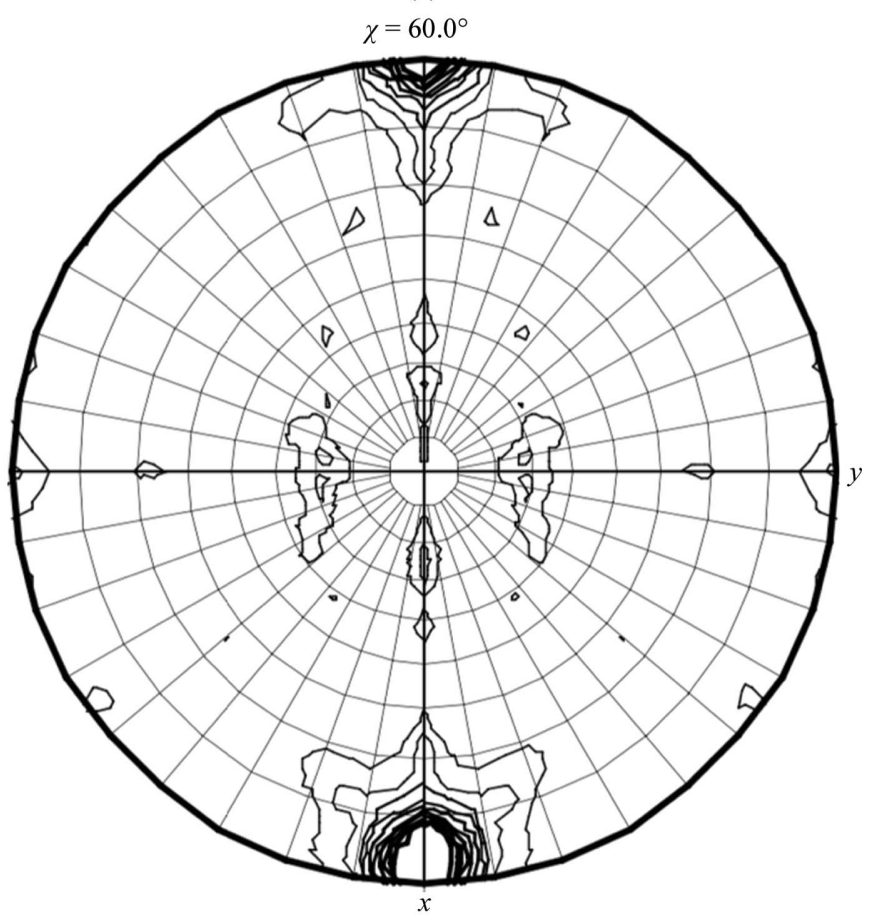

(c)

Figure 1

Purification and self-rotation function analysis of Rho. (a) Size-exclusion chromatograph of NaAtm1 purification using a HiLoad Superdex 200 16/60 column. The column void volume is colored gray, while the elution positions of various molecular-weight standards (in $\mathrm{kDa}$ ) are marked on the chromatograph. (b) SDS-PAGE of the peak fractions of SEC purification from $(a)$. (c) The $\chi=180^{\circ}$ and $60^{\circ}$ sections of the self-rotation function calculated using MOLREP in CCP4 (Winn et al., 2011) with an integration radius of $51 \AA$ using diffraction data between 3.3 and $40 \AA$ resolution. 
subunit and a corresonding translation along the axis of $-7.7 \AA$. In comparison, the corresponding values for PDB entry 1 pvo are $57.9^{\circ}$ and $-8.2 \AA$, respectively. Reflecting the larger rotation angle per subunit, the present structure exhibits a more closed ring in comparison to the original brokenstaircase structure (Figs. $3 a$ and $3 b$ ). At the level of subunitsubunit interactions, however, the differences are subtle and we could not identify the specific interactions responsible for these differences in hexamer structure. Of note, the nucleotidebinding site is at the interface between subunits and small changes in subunit-subunit interactions may reflect the presence of different nucleotides: ATP in this structure and either no nucleotide (apo), ATP $\gamma \mathrm{S}$ or AMPPNP in other open-ring structures (Skordalakes et al., 2005; Skordalakes \& Berger, 2003).
How did Rho end up in our crystallization conditions? Our hypothesis is that during the overexpression of proteins the E. coli transcription and translation machineries are highly expressed for the production of mRNAs and recombinant proteins, respectively. Rho, as the termination factor, would plausibly be overexpressed as part of the transcriptiontermination process; thus, it is likely that Rho is a general contaminant and does not arise specifically from the overexpression of $\mathrm{NaAtm} 1$. The fact that Rho eluted from the $\mathrm{Ni}-$ NTA column along with His-tagged NaAtm1 suggests that there is nonspecific binding to the $\mathrm{Ni}^{2+}$ resin by the histidine residues distributed throughout the whole protein (Fig. 3c). The open-ring conformation that Rho adopts in solution (Thomsen et al., 2016) with a molecular weight of $282 \mathrm{kDa}$ for the hexamer apparently has a comparable hydrodynamic
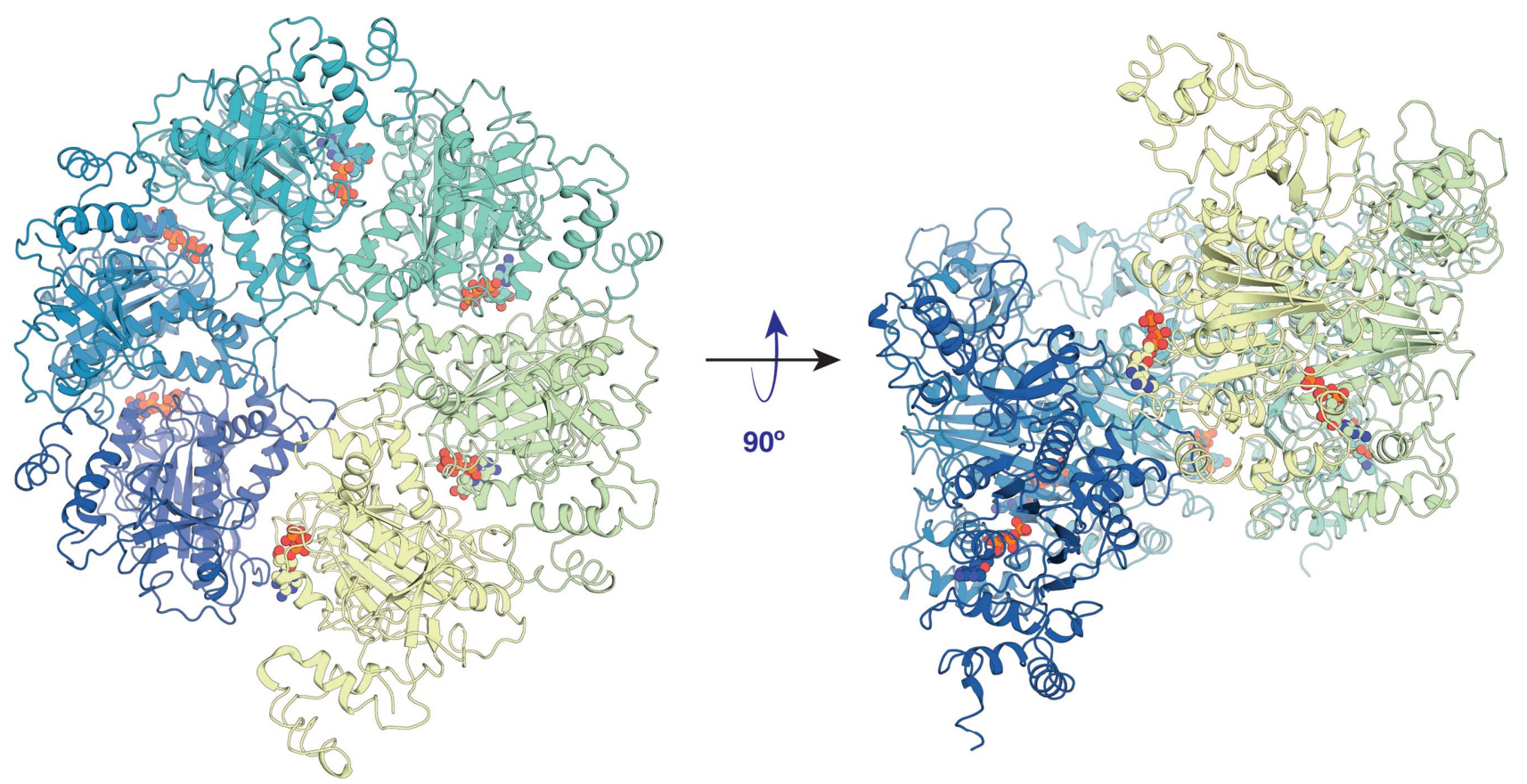

(a)
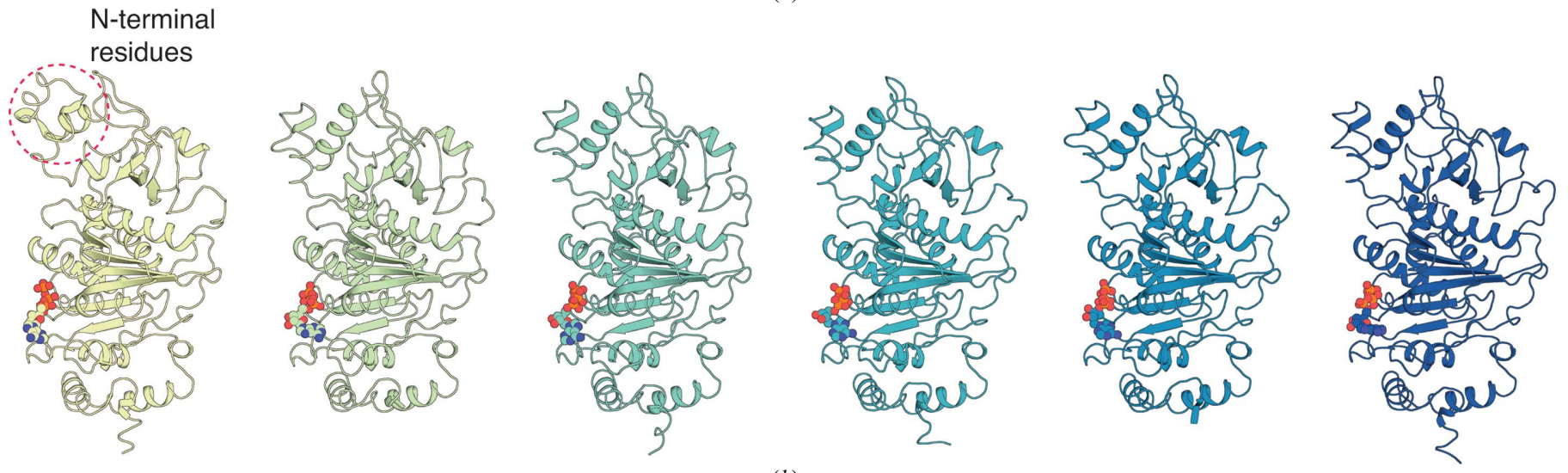

Figure 2

Crystal structure of Rho. (a) Overall view of Rho in broken-staircase conformation with ATP bound. (b) Single subunit of Rho with ATP bound. The six subunits are shown separately and ATP is shown as red spheres. The dashed circle identifies the N-terminal $\sim 50$ residues of the Rho subunit positioned at the break in the hexameric staircase arrangement; this region has rearranged in this subunit relative to the conformation exhibited in the other five subunits. 
radius to $\mathrm{NaAtm} 1$, with a dimer molecular weight of $133 \mathrm{kDa}$ in addition to the detergent micelle. Given the low abundance, the presence of Rho in the SEC fractions was only detected in hindsight. Also in hindsight, Rho was not present in the original NaAtm1 purification, which included a membraneisolation step in which Rho was presumably removed (Lee et al., 2014); in the present work the membrane-isolation step was omitted and Rho subsequently copurified with NaAtm1.

We have also observed Rho in single-particle cryoEM studies of NaAtm1 reconstituted in membrane-scaffolding protein (MSP) nanodiscs. The NaAtm1 nanodisc sample was prepared by incubating detergent-purified NaAtm1 with MSPs and lipids, and was further purified by size-exclusion chromatography. Similar to the purification in detergent, the peak fractions were collected for single-particle cryoEM sample preparation (Fig. 4a). The 2D classification reported one class of Rho in the broken hexameric state (Fig. $4 b$ ), again suggesting that Rho has a similar hydrodynamic radius and plausibly a similar molecular weight to our reconstituted NaAtm1 in nanodiscs.

In a structural analysis of a prokaryotic chloride channel, a single peptide of Rho was identified in a mass-spectrometric analysis of the gel band (Abeyrathne \& Grigorieff, 2017), representing the first time, to our knowledge, that Rho has been identified as a possible contaminant during membraneprotein expression. As demonstrated in this report, Rho can

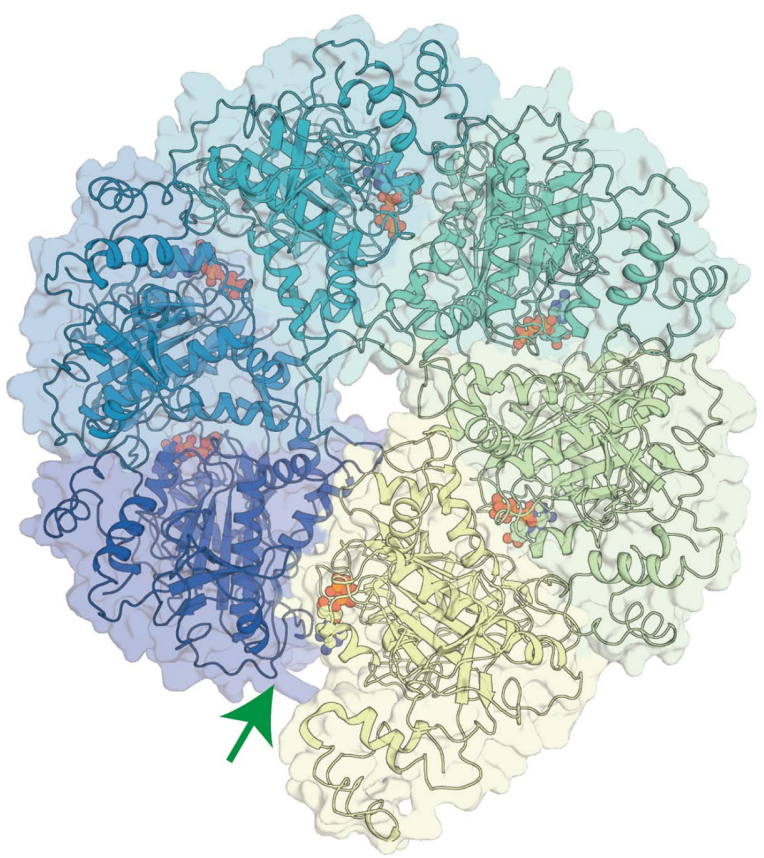

(a)
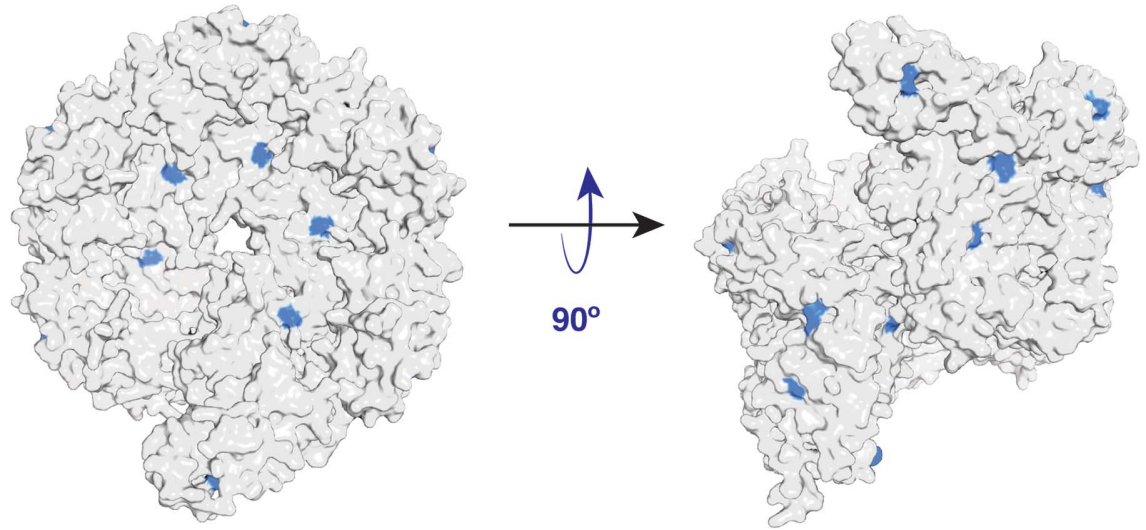

$(c)$

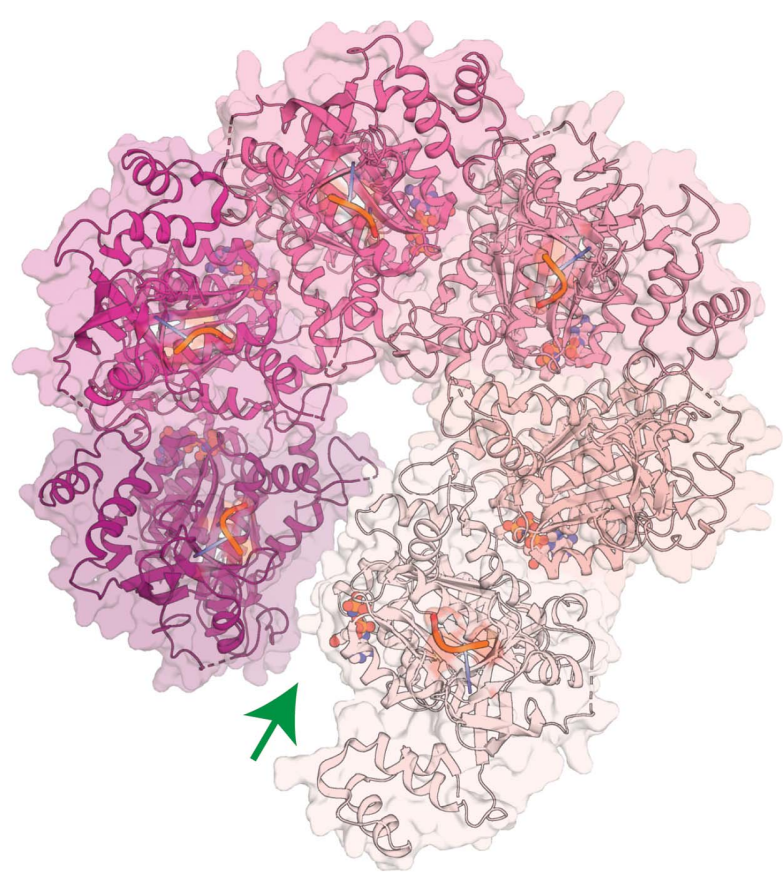

(b)

Figure 3

Overall architecture of Rho. Overall structural representations of ( $a$ ) ATP-bound Rho (this study) and (b) the AMPPNP-bound structure of Rho (PDB entry 1pvo; Skordalakes \& Berger, 2003). (c) Distribution of surface histidine residues (blue) in the ATP-bound structure of Rho (this work). The spacings between the surface-exposed histidines are several nanometres and are comparable to the loading density of His-tagged proteins bound to NiNTA beads (Hayworth \& Hermanson, 2014), which presumably allows multiple binding sites to Ni-NTA and contributes to the observed affinity of Rho for Ni-NTA resin. 


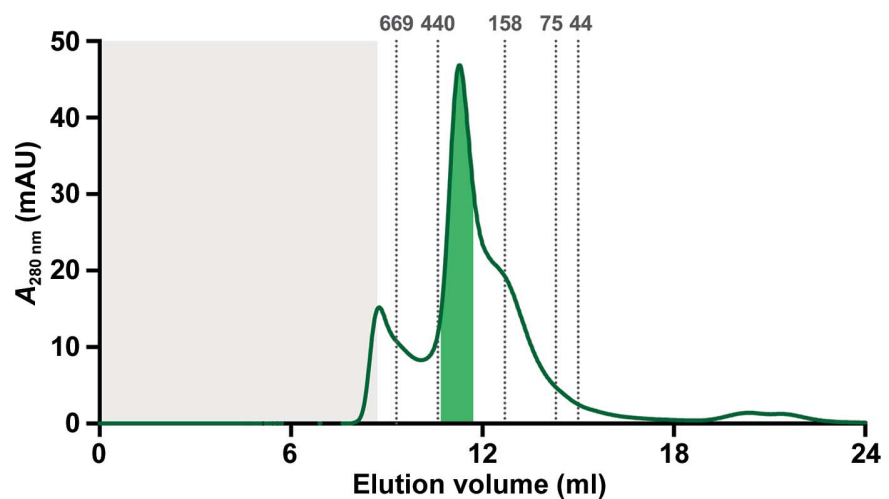

(a)
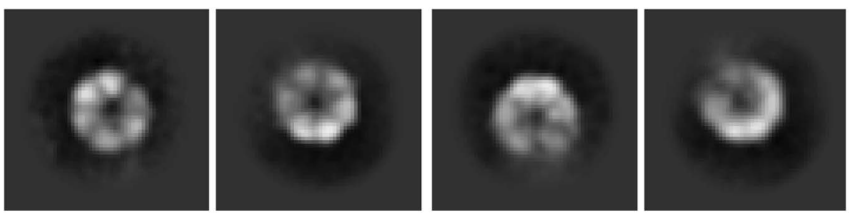

(b)

Figure 4

Single-particle cryo-EM analysis of $\mathrm{NaAtm} 1$ samples containing Rho. (a) Size-exclusion chromatograph of the reconstituted $\mathrm{NaAtm} 1$ in MSP1D1 nanodiscs using a Superdex 200 Increase 10/300 column. The column void volume is colored gray, while the elution positions of various molecularweight standards (in $\mathrm{kDa}$ ) are marked on the chromatograph. (b) 2D classes of Rho illustrating the hexameric arrangement with a diameter of $\sim 100 \AA$. The box size of the $2 \mathrm{D}$ classes is $284 \times 284 \AA$.

crystallize even in the presence of a large excess of other proteins, and thus it should be added to the list of known contaminant proteins in crystallography.

\section{Acknowledgements}

We thank Jens T. Kaiser and the beamline staff at Advanced Photon Source GM/CA beamline 23-ID-B and Stanford Synchrotron Radiation Lightsource (SSRL) beamline 12-2 for their support during crystallographic data collection, Mona Shahgholi at the Caltech CCE Multiuser Mass Spectrometry Laboratory for Rho identification, and Andrey Malyutin and Songye Chen at the Caltech CryoEM facility for their support during electron-microscopy data collection. We gratefully acknowledge the Gordon and Betty Moore Foundation and the Beckman Institute at Caltech for their generous support of the Molecular Observatory at Caltech. Cryo-electron microscopy was performed in the Beckman Institute Resource Center for Transmission Electron Microscopy at Caltech. GM/ CA@APS has been funded in whole or in part with Federal funds from the National Cancer Institute (ACB-12002) and the National Institute of General Medical Sciences (AGM12006). This research used resources of the Advanced Photon Source, a US Department of Energy (DOE) Office of Science User Facility operated for the DOE Office of Science by Argonne National Laboratory under Contract No. DE-AC0206CH11357. The EIGER 16M detector was funded by an NIH-Office of Research Infrastructure Programs High-End Instrumentation Grant (1S10OD012289-01A1). Use of the Stanford Synchrotron Radiation Lightsource, SLAC National
Accelerator Laboratory is supported by the US Department of Energy, Office of Science, Office of Basic Energy Sciences under Contract No. DE-AC02-76SF00515. The SSRL Structural Molecular Biology Program is supported by the DOE Office of Biological and Environmental Research and by the National Institutes of Health, National Institute of General Medical Sciences (including P41GM103393).

\section{Funding information}

The following funding is acknowledged: Howard Hughes Medical Institute.

\section{References}

Abeyrathne, P. D. \& Grigorieff, N. (2017). PLoS One, 12, e0180163. Das, D., Xu, Q. S., Lee, J. Y., Ankoudinova, I., Huang, C., Lou, Y., DeGiovanni, A., Kim, R. \& Kim, S.-H. (2007). J. Struct. Biol. 158, 494-502.

Emsley, P., Lohkamp, B., Scott, W. G. \& Cowtan, K. (2010). Acta Cryst. D66, 486-501.

Evans, P. R. \& Murshudov, G. N. (2013). Acta Cryst. D69, 12041214.

Hayworth, D. A. \& Hermanson, G. T. (2014). Calculate the Number of Immobilized Proteins Per Bead of Agarose Affinity Supports. https:// www.thermofisher.com/us/en/home/life-science/protein-biology/ protein-biology-learning-center/protein-biology-resource-library/ protein-biology-application-notes/calculate-number-immobilizedproteins-per-bead-agarose-affinity-supports.html.

Hungler, A., Momin, A., Diederichs, K. \& Arold, S. T. (2016). J. Appl. Cryst. 49, 2252-2258.

Kabsch, W. (2010). Acta Cryst. D66, 125-132.

Lee, J. Y., Yang, J. G., Zhitnitsky, D., Lewinson, O. \& Rees, D. C. (2014). Science, 343, 1133-1136.

Liebschner, D., Afonine, P. V., Baker, M. L., Bunkóczi, G., Chen, V. B., Croll, T. I., Hintze, B., Hung, L.-W., Jain, S., McCoy, A. J., Moriarty, N. W., Oeffner, R. D., Poon, B. K., Prisant, M. G., Read, R. J., Richardson, J. S., Richardson, D. C., Sammito, M. D., Sobolev, O. V., Stockwell, D. H., Terwilliger, T. C., Urzhumtsev, A. G., Videau, L. L., Williams, C. J. \& Adams, P. D. (2019). Acta Cryst. D75, 861-877.

Nam, K. H., Xu, Y., Piao, S., Priyadarshi, A., Lee, E. H., Kim, H.-Y., Jeon, Y. H., Ha, N.-C. \& Hwang, K. Y. (2010). Biochem. Biophys. Res. Commun. 391, 990-994.

Niedzialkowska, E., Gasiorowska, O., Handing, K. B., Majorek, K. A., Porebski, P. J., Shabalin, I. G., Zasadzinska, E., Cymborowski, M. \& Minor, W. (2016). Protein Sci. 25, 720-733.

Punjani, A., Rubinstein, J. L., Fleet, D. J. \& Brubaker, M. A. (2017). Nat. Methods, 14, 290-296.

Ritchie, T. K., Grinkova, Y. V., Bayburt, T. H., Denisov, I. G., Zolnerciks, J. K., Atkins, W. M. \& Sligar, S. G. (2009). Methods Enzymol. 464, 211-231.

Rohou, A. \& Grigorieff, N. (2015). J. Struct. Biol. 192, 216-221.

Simpkin, A. J., Simkovic, F., Thomas, J. M. H., Savko, M., Lebedev, A., Uski, V., Ballard, C., Wojdyr, M., Wu, R., Sanishvili, R., Xu, Y., Lisa, M.-N., Buschiazzo, A., Shepard, W., Rigden, D. J. \& Keegan, R. M. (2018). Acta Cryst. D74, 595-605.

Skordalakes, E. \& Berger, J. M. (2003). Cell, 114, 135-146.

Skordalakes, E., Brogan, A. P., Park, B. S., Kohn, H. \& Berger, J. M. (2005). Structure, 13, 99-109.

Stepanov, S., Makarov, O., Hilgart, M., Pothineni, S. B., Urakhchin, A., Devarapalli, S., Yoder, D., Becker, M., Ogata, C., Sanishvili, R., Venugopalan, N., Smith, J. L. \& Fischetti, R. F. (2011). Acta Cryst. D67, 176-188.

Thomsen, N. D. \& Berger, J. M. (2009). Cell, 139, 523-534. 
Thomsen, N. D., Lawson, M. R., Witkowsky, L. B., Qu, S. \& Berger, J. M. (2016). Proc. Natl Acad. Sci. USA, 113, E7691E7700.

Veesler, D., Blangy, S., Cambillau, C. \& Sciara, G. (2008). Acta Cryst. F64, 880-885.
Winn, M. D., Ballard, C. C., Cowtan, K. D., Dodson, E. J., Emsley, P., Evans, P. R., Keegan, R. M., Krissinel, E. B., Leslie, A. G. W., McCoy, A., McNicholas, S. J., Murshudov, G. N., Pannu, N. S., Potterton, E. A., Powell, H. R., Read, R. J., Vagin, A. \& Wilson, K. S. (2011). Acta Cryst. D67, 235-242. 